\title{
Intention to Seek Regular HIV and Syphilis Testing and Associated Factors Among Female Sex Workers in Uganda
}

Richard Muhindo ( $\nabla$ r.muhindo@yahoo.com )

Makerere University College of Health Sciences https://orcid.org/0000-0002-3249-117X

Barbara Castelnuovo

Makerere University Infectious Diseases Institute

Andrew Mujugira

Makerere University Infectious Diseases Institute

Nelson K. Sewankambo

Makerere University School of Medicine

\section{Rosalind Parkes-Ratanshi}

University of Cambridge Institute of Public Health

Juliet Kiguli

School of Public Health Makerere University

Nazarius Mbona Tumwesigye

School of Public Health Makerere University

Edith Nakku-Joloba

School of Public Health Makerere University

\section{Research article}

Keywords: HIV; Syphilis; Testing; Female Sex Workers; Africa

Posted Date: March 3rd, 2020

DOI: https://doi.org/10.21203/rs.3.rs-15682/v1

License: (1) (1) This work is licensed under a Creative Commons Attribution 4.0 International License. Read Full License 


\section{Abstract}

Background: Few studies have evaluated intentions to undergo periodic sexually transmitted infection (STI) and HIV testing among female sex workers (FSW) in Sub-Saharan Africa. We aimed to assess intention to seek periodic syphilis and HIV testing among FSW in Uganda.

Methods: Between July and October 2018, we conducted a cross-sectional study among 441 FSW. Participants were recruited through peer referrals. Self-reported data on intention to take a syphilis test in the next 3 months and an HIV test in next 6 months were obtained using an interviewer-administered questionnaire. We used constructs of the Integrated Change Model to assess intentions, attitudes, norms, social influences and self-efficacy towards 3-monthly syphilis and 6-monthly HIV testing. Predictors of intention to seek testing were estimated using linear regression.

Results: A total of $441 \mathrm{FSW}$ were included in the analysis, and the median age was 26 years (interquartile range [IQR] 23-30). Most (66.9\%) reported high intention to take an HIV test in the next 6 months, and $51.9 \%$ showed high intention to take a serological syphilis test in the next 3 months. In multivariable analysis, never testing for HIV was associated with low intention of testing $(\beta=-0.15, p=0.001)$. Factors significantly associated with high intentions to test for HIV in the next 6 months were perceived influence of significant others $(\beta=0.08, p=0.03)$, high self-efficacy $(\beta=0.26, p=0.001)$ and high attitude scores $(\beta=0.45, p=0.001)$. Similarly, high attitude $(\beta=0.30, P=0.001)$ and descriptive norms $(\beta=0.32, P=0.001)$ were associated with high intentions to test for syphilis in the next three months.

Conclusion: These results suggest public health programs promoting routine HIV and syphilis testing should consider factors such as attitudes, descriptive norms, social influences, self-efficacy and past behaviours which inform intention to undergo STI testing.

\section{Background}

Uganda has registered significant strides in the fight against HIV. Between 2011 and 2017, adult HIV prevalence declined from $7.3 \%$ to $5.9 \%$, respectively $[1,2]$. The number of new HIV infections decreased from 123,803 in 2013 to 47,469 in 2017, a 62\% decline [3]. Despite these successes, Uganda still ranks among the countries contributing new HIV infections in Eastern and Southern Africa (ESA) [4]. Of the 800,000 new HIV infections which occurred in the region in 2017, nearly 70\% were contributed by Kenya, Mozambique, South Africa, Tanzania and Uganda [4]. Sex work is a major driver of the HIV epidemic in ESA: female sex workers (FSW) and their clients accounted for $10 \%$ of new HIV infections in 2017 [4]. In Uganda, $7-11 \%$ of new HIV infections are attributable to sex work [5]. The estimated HIV prevalence among FSW is $32.4-52 \%$ [6, 7], compared with $7.3 \%$ among adult women in the general population [1]. The high HIV burden among Ugandan FSW is associated with co-infection with other sexually transmitted infections (STI) [6]. Studies have found high burden of STIs among FSW in Uganda, including Herpes Simplex Virus type-2 [HSV-2] (80\%), Neisseria gonorrhoeae (13\%) and Chlamydia trachomatis (9\%) [6, 8, 9]. Concurrent STI infection increases HIV acquisition and transmission risk [10-12]. 
The World Health Organization (WHO) recommends 3-monthly STI testing, and 6-monthly HIV testing, for FSW $[13,14]$. Regular testing, an essential step to knowing one's status and obtaining prevention, treatment and care services, has individual and public health benefits [15-17]. These include linkage to HIV care and reduced risky sexual behaviors $[17,18]$; suppressive antiretroviral treatment (ART) decreases transmission risk by 95\% [18]. However, a significant proportion of Ugandan FSWs are unaware of their HIV status. Only $53 \%$ reported ever testing for HIV, and $16 \%$ had tested in the prior 12 months [6] despite availability of HIV testing services (HTS) including targeted moonlight testing approaches [19, 20]. Prior studies have shown that uptake of HTS is hindered by individual, structural, societal and policy-level barriers $[19,21]$. However, few studies have explored motivations influencing uptake of HTS among FSW in Uganda.

Regular testing is a rational behavior that may largely be influenced by behavioral intentions. Intention is a major determinant of health behavior including participation in health screening programs [22-25]. Intention or motivation to adopt STI and HIV testing by FSW on a regular basis may be influenced by personal beliefs, knowledge, experiences, social environment, quality of services and quality of interaction with service providers, which interact to predict FSW motivation to adopt regular STI and HIV testing behavior $[19,26]$. In this study we sought to determine factors associated with FSW intention to seek syphilis and HIV testing in the next 3 and 6 months, respectively.

\section{Methods}

\section{Participants and procedures}

Between July and October 2018, we conducted a cross-sectional survey to describe regular STI and HIV screening intentions among FSW in the municipalities of Arua, Kampala, Mbale, Mbarara (combined population 1,857,088) [27] located in the Northern, Central, Eastern and Western regions of Uganda, respectively. Prior to participant recruitment, a mapping exercise was conducted to gain an understanding of the time sex work occurs, hot spots, typologies, sex work procurer connections and territorial management in each municipality. The main typologies based on venue were street, lodge, and bar/club, and brothel (mainly in Kampala). Sex work hotspots constituted the primary sampling units (PSUs). Based on the different typologies, lists were drawn to form a sampling frame for each municipality. In Kampala, we randomly selected 20 PSUs, consisting of brothels, lodges, streets and bars/clubs. In Arua, Mbale and Mbarara, we randomly selected between 10-15 PSUs consisting mainly of lodges, street and bars/clubs. Study participants included women $\geq 17$ years engaged in sex work according to self-report of selling sex for goods or money for at least 6 months. Between 7 and 10 participants were recruited from each PSU in each municipality.

We used a two-stage sampling design to recruit study participants. Sampling began by identifying 2-3 FSWs at each PSUs as peer recruiters; these were selected through key informants (bar maids, pimps and managers). Recruiters were given a brief training on peer recruitment and provided with 2-3 paper coupons. Each coupon contained an identification number, contact information of the research team and 
the duration of the survey in the municipality. Only participants who presented the coupon after verification, and met the eligibility criteria, were asked to provide informed consent to participate in an interviewer-administered questionnaire. The questionnaire was administered by trained research assistants at a venue agreed upon with the participant. All study participants received information about STI and HIV screening during the consenting process and after the interview.

\section{Data collection}

A 65-item questionnaire that was validated among FSW in Benin [28] was used to assess intention (readiness) to take a syphilis serological test (INT-SYP) in the next 3 months and an HIV (INT-HIV) test in the next 6 months by asking respondents three items for each infection, e.g., "Are you going to be screened for syphilis in the next 3 months"? Answering options ranged from $1=$ 'Strongly disagree' to $6=$ 'Strongly agree' (Cronbach's $a=0.94$ for INT-SYP and 0.90 for INT-HIV). The main independent variables derived from the constructs of the Integrated Change Model (I-Change) [22] were attitude (ATT), social norms (SN), self-efficacy (SE) and descriptive norms (DN).

\section{Attitude}

Attitudes towards 3-monthly syphilis testing (ATT-SYP) were assessed through three items asking respondents to what extent they agreed with statements such as, "For you getting tested for syphilis every 3 months, would reduce your risk of contracting HIV" (Cronbach's a $=0.7$ ). Answering options on a 6-point scale ranged from 1 = 'Strongly disagree' to $6=$ 'Strongly agree'. Attitudes towards 6 -monthly HIV testing (ATT-HIV) were assessed by asking respondents five items about the benefits and disadvantages of being tested for HIV every 6 months (Cronbach's a $=0.75$ ).

\section{Descriptive norms}

Descriptive norms regarding 3-monthly syphilis testing (DN-SYP) were assessed by asking respondents three questions about whether FSW thought it was their moral obligation to test and how often they thought their peers were testing for syphilis every 3 months. Respondents were asked to what extent they agreed with statements like "Being tested for syphilis is a normal routine that many FSWs practice?" Answering options on a 6-point scale ranged from $1=$ 'Strongly disagree' to $6=$ 'Strongly agree' (Cronbach's $a=0.7$ ). Three items on the same scale were used to assess descriptive norms to 6-monthly HIV testing (DN-HIV) (e.g. "based on what you know about your fellow FSWs and the practice of HIV testing, how many of them are being tested every six months. Answering options ranged from $1=$ 'None to $6=$ 'All' (Cronbach's a $=0.7)$.

\section{Self-efficacy}


Self-efficacy for 6-monthly HIV testing (SE-HIV) was assessed by seven questions about their perceived level of confidence and ease of seeking 6-monthly HIV testing. Items included a range of barriers including stigma, discrimination, fear of positive results, privacy and confidentiality. Questions included, "Do you feel able to go for an HIV test every six months, even if you are afraid of receiving a positive result?" (Cronbach's a $=0.7$ ). Answering options on a 6 -point scale ranged from $1=$ 'Strongly disagree' to $6=$ 'Strongly agree'.

\section{Social norms}

Social norms regarding 6-monthly HIV testing (SN-HIV) were assessed on a 6-point scale (1 = 'Disapprove strongly to $6=$ 'Approve strongly') by asking respondents two questions on whether referent others (fellow FSWs and regular partners/clients) approved or expected them to test for HIV every six months (Cronbach's alpha $=0.66$ ).

\section{Statistical analysis}

The primary outcome was intention to take a syphilis serological test (INT-SYP) in the next 3 months and an HIV test (INT-HIV) in the next 6 months. We used Cronbach's alpha to assess scale reliability and compute scale medians [29]. Principal component analysis (PCA) was used to reduce 29 items in the questionnaire related to intention, attitudes, social norms, descriptive norms and self-efficacy and establish any underlying structure. We used the Kaiser-Meyer-Olkin measure to verify sampling adequacy [30]. Bartlett's test of sphericity indicated that correlations between the items were large enough for PCA [31]. Using Cronbach's a statistic, we assessed the reliability of item scales and computed scale medians. Participants were considered to score high on the item scale, if their score was above the scale median. Parametric tests were used to analyse Likert-type scale ordinal data [32, 33]. A multiple linear regression model was used to evaluate potential factors (age, level of education, marital status, town location, previous testing behavior, attitude, social influences, descriptive norm, and self-efficacy) associated with intention to undertake syphilis and HIV testing. Data analyses were performed using SPSS version 23.0 (IBM Corp, Armonk, NY) and Stata version 12.0 (StataCorp, College Station, TX).

\section{Ethical approval}

Ethical clearance for the study was obtained from the Higher Degrees, Research and Ethics Committee, School of Public Health, Makerere University and Uganda National Council for Science and Technology (HS 2403). All respondents provided written consent in English or their local language. To protect human subjects, all members of the research team completed a research ethics course with emphasis on vulnerable populations. All interviews were conducted at places agreed with participants and no personal identifiers were obtained. No financial payments were made to pimps, lodge and bar managers to avoid coercion in recruitment. 


\section{Results}

\section{Socio-demographic characteristics}

A total of $441 \mathrm{FSW}$ took part in the study and the median age was 26 years (interquartile range [IQR] 2330) (Table 1). Just over half (52.8\%) of the participants had obtained primary education and $10.2 \% \mathrm{had}$ no formal education. The median duration of sex work was 32 months (IQR 17-60) and common venues of operation were lodges (36.0\%), streets (32.8\%), bars/clubs (23.5\%) and brothels (3.4\%). A majority $(82.1 \%)$ were biological mothers, and most $(58.7 \%)$ reported consistent condom use during work. Of the $77.3 \%$ who reported ever being in a relationship (either married or having a boyfriend), $42.4 \%$ had separated from their partner. Nearly all (95\%) reported ever testing for HIV and just over half (59\%) reported ever testing for syphilis.

\section{HIV and syphilis testing}

Two-thirds (66.9\%) had high intention to undergo an HIV test in the next 6 months, whereas $48.1 \%$ had low intention to test for syphilis in the following 3 months (Table 2). Preferred venues for testing were public health facility (55.6\%), private clinic (39\%), outreach (5\%) and non-governmental health facility (0.5\%). The median score for attitude towards 6 monthly HIV testing was 21 (IQR 20-22) with just over half $(52.4 \%)$ of respondents posting high attitude scores. Most respondents (59.2\%) thought that 6 monthly HIV testing was a common practice among other FSW, with a significant proportion (48.5\%) reporting that fellow FSW and or their clients would disapprove 6-monthly HIV testing. Self-efficacy was modestly high, with $57.6 \%$ believing they could confidently undergo 6-monthly HIV testing at health clinics despite barriers related to fear of positive results, stigma and discrimination, lack of privacy, confidentiality and transport costs. The median self-efficacy score for 6-monthly HIV testing was 29 (IQR 26-32). A similar proportion of respondents (50.6\%) scored high on attitudes towards 3 monthly syphilis testing with a median score of 9 (IQR, 6-10) (Table 3). Most respondents (51.5\%) thought 3-monthly syphilis testing was not a common practice among fellow FSW. However, a significant proportion (59.2\%) believed they could confidently test for syphilis every 3 months.

\section{Associations with HIV and syphilis testing}

A multivariable linear regression analysis identified I-Change Model constructs that predicted the likelihood of intention to test for HIV in the next six months (Table 4). The model showed a significant proportion of variation in intention to test for HIV in the next six months $\left(R^{2}=0.51 ; F(19.42)=34.2\right.$; $\mathrm{p}$ <0.001). After adjusting for socio-demographic factors (age, level of education, town location, and marital status), condom use practices and descriptive norms, the structural model showed that $61 \%$ of the variance in intention was explained by previous testing behavior, social influences, self-efficacy and attitudes in ascending order of importance (standardized coefficient weight, $\beta$ ). Among the respondents, having never tested for HIV was associated with low intention $(\beta=-0.15, p=0.001)$. Factors associated 
with high intentions to test for HIV in the next 6 months were perceived influence of significant others $(\beta=$ $0.08, p=0.03)$, high self-efficacy $(\beta=0.26, p=0.001)$ and attitude scores $(\beta=0.45, p=0.001)$.

The model also showed a significant proportion of variation in intention to test for syphilis in the next three months $\left(R^{2}=0.51 ; F(18.42)=24.0 ; p<0.001\right)$. After adjustment for socio-demographics (age, level of education, and marital status), condom use practices and self-efficacy, the structural model showed that $51 \%$ of the variance in intention was explained in ascending order of importance (standardized coefficient weight, $\beta$ ) by previous testing behavior, town location, attitude and descriptive norms (Table 5). High attitude $(\beta=0.30, P=0.001)$ and descriptive norm scores $(\beta=0.32, P=0.001)$ were associated with high intentions to test for syphilis in the next three months. By contrast, having never tested for syphilis was associated with low intention $(\beta=-0.21, p=0.001)$, particularly among respondents from Mbarara when compared to Kampala $(\beta=-0.07, p=0.046)$.

\section{Discussion}

In this cross-sectional study of $441 \mathrm{FSW}$ in Uganda, syphilis testing was less frequent than HIV testing. Half of the participants had high intentions to undergo serological syphilis testing in the next 3 months compared with two-thirds intending to test for HIV in the next 6 months. Predictors of intention to undergo syphilis testing were previous testing behavior, geographical location, attitude, and descriptive norms. By contrast, social influences, self-efficacy and attitudes were associated with intentions to take an HIV test in the next 6 months.

Our finding that nearly all FSW (95\%) reported ever testing for HIV test is higher than the proportion (55\%) previously reported in this setting [6], but consistent with findings from Kenya, Benin and Rwanda, where $86.6 \%, 87.1 \%$ and $90.0 \%$ of FSW, respectively, reported ever testing for HIV [28, 34, 35]. The higher testing rates we found could be due to targeted HIV testing programs for FSW in Uganda which aim to achieve the national strategic plan target of at least $90 \%$ testing coverage among persons with HIV in order to achieve HIV epidemic control by $2030[19,36]$.

We observed that FSW had low intention to regularly test for syphilis, perhaps because of limited knowledge of the benefits of periodic STI testing. Behavioural intentions are reported to explain up to 30$50 \%$ of the variance in health seeking behaviours including STI testing [22-24,37]. Studies have found that negative attitudes, low perceived benefits and lack of knowledge of screening guidelines are associated with low screening intentions [28, 38-42]. However, none of these studies evaluated periodic screening intentions among FSW for STIs other than HIV. Additionally, public health programs in this setting mostly emphasize HIV testing for FSWs $[19,28,43]$ perhaps because of funding priorities. Our findings suggest that programs should also emphasise periodic testing for syphilis and other STIs. Uganda guidelines now recommend dual syphilis and HIV testing for pregnant women and their partners in order to prevent mother-to-child transmission of syphilis and HIV [44]. Our findings suggest that this approach should be extended to FSW. 
We found that attitudes and previous testing behaviour were significantly associated with high intention to undertake syphilis and HIV testing. Similar findings have been demonstrated in studies of health behavioural intentions, but most of these studies evaluated cervical cancer screening or physical activity in populations other than FSW $[28,38-40,42]$. Previous testing history may influence testing attitudes by overcoming fears and misconceptions of STI screening. We observed that five-in-ten FSW had negative attitudes towards 3-monthly syphilis testing compared to four-in-ten for 6-monthly HIV testing. Attitudes are influenced by knowledge and personal evaluation of the positive and negative consequences of periodic STI testing $[22,37]$. The high proportion of negative attitudes we observed could be due to lack of comprehensive knowledge about the benefits of periodic STI screening or barriers to testing including fear, stigma and discrimination of sex workers. Lack of knowledge and perceived negative consequences are barriers to testing, whereas knowledge of the benefits of STI testing and past testing behaviours facilitate testing uptake $[45,46]$. Awareness of the testing behaviour of fellow FSW appears to be an important factor in normalizing periodic testing behaviours [28, 43].

We found that prior testing history significantly predicted intention to test which was probably mediated through attitudes, self-efficacy and social influences. Most FSW who scored high on self-efficacy to undergo periodic HIV testing amidst barriers (stigma, fear of positive results, lack of privacy and confidentiality) were likely to have high intention to test for HIV in the next 6 months. Self-efficacy may help overcome internal stigma associated with sex work and HIV. Our findings are consistent with prior work in which self-efficacy, social influences, attitudes and past behaviour were predictors of intention to test $[47,48]$. Notably, self-efficacy and social influences were significantly positively associated with intention to test for HIV but not syphilis. This could be due to the differences in knowledge about the two infections and relative perception of their overall effect on the life of an FSW. Culturally, syphilis is perceived as a sign of virility and is less stigmatized than HIV [49]. This could help explain why descriptive norms (perceived prevalence of behaviour among peers) were significantly positively associated with intention to test for syphilis but not HIV. Intention to test for syphilis could be modulated through attitudes and descriptive norms while HIV testing intention is modulated through attitudes, social influences and self-efficacy. Thus, public health programs should take into account these differences when developing messages that promote routine syphilis and HIV testing for sex workers.

The strengths of our study include nationally representative sampling and use of a conceptual model to guide study design and analysis. The limitations of this cross-sectional study include the inability to determine temporal relationships between exposures and outcomes. Recall and social desirability bias could have influenced self-reported syphilis and HIV testing behaviors. However, we used constructs of the I-change model to guide the design of the questionnaire and exploratory factor analysis using principal component analysis on validity and reliability of question items during analysis.

\section{Conclusions}

This study operationalized the Integrated Change Model to explain intentions to seek syphilis and HIV testing among FSW in Uganda. We found that attitudes, descriptive norms and past behaviour were 
predictive of intention to test for syphilis while attitudes, social influences, self-efficacy and past behaviour were associated with intention to undergo HIV testing. These findings suggest that public health programs should consider these factors when designing messaging promoting routine testing for syphilis and HIV.

\section{Declarations}

\section{Abbreviations}

FSW: female sex workers; HIV: human immunodeficiency virus; IQR: interquartile range; PCA: principal component analysis; PSU: primary sampling unit; STI: sexually transmitted infection; WHO: World Health Organization.

\section{Acknowledgements}

Richard Muhindo is a NURTURE fellow funded through grant D43TW010132 from the National Institutes of Health. Special thanks to NURTURE program, mentors and the secretariat. He is also a PhD scholar under the Johnson \& Johnson Corporate Citizenship Trust grant at Ugandan Academy of Health Innovation and Impact, Infectious Diseases Institute Uganda. Special thanks to the Infectious Diseases Institute (IDI), PhD scholar Mentorship program and the IDI PhD scholar forum.

The authors thank the leadership of Kampala Capital City Authority and the municipalities of Arua, Mbarara and Mbale which enabled the data collection process. We thank the participants for volunteering to participate in the study. We thank Dr. Albino Kalolo, Dr. Van Hennik Matthea and Mr. Moses Ray Ogwang for accepting to proofread the manuscript during the development phase. Finally, we thank Collins Twesigye, Jennifer Bako, Alfred Okuonzi and Michael Muhoozi who worked as research assistants.

\section{Author contributions}

RM conceived the research idea, participated in the design of the study including coordination of data collection, and drafting of the manuscript. BC, RP, NK, JK, NM, AM, NKS and EN, all participated in refining the research idea, design including tools. RM, EN and NM participated in the statistical data analysis. RM, $\mathrm{BC}$, and $\mathrm{AM}$ wrote the first draft. All authors contributed to interpretation of the results and the writing of the manuscript, and all approved the final manuscript.

\section{Funding}

This study was supported through grant number D43TW010132 supported by Office Of The Director, National Institutes Of Health (OD), National Institute Of Dental \& Craniofacial Research (NIDCR), National 
Institute Of Neurological Disorders And Stroke (NINDS), National Heart, Lung, And Blood Institute (NHLBI), Fogarty International Center (FIC), National Institute On Minority Health And Health Disparities (NIMHD) and a grant from the Ugandan Academy of Health Innovation and Impact. The Ugandan Academy is initially funded by Janssen, the Pharmaceutical Companies of Johnson \& Johnson as part of its commitment to global public health through collaboration with the Johnson \& Johnson Corporate Citizenship Trust. AM was supported through grant K43TW010695 from the National Institutes of Health.

\section{Availability of data and materials}

The datasets used during the current study are available from the corresponding author on request. The questionnaire is included as supplementary information.

\section{Ethics approval and consent to participate}

Ethical clearance for the study was obtained from the Higher Degrees, Research and Ethics Committee, School of Public Health, Makerere University and Uganda National Council for Science and Technology (HS 2403). All respondents provided written consent in English or their local language.

\section{Competing interests}

The author(s) declare that they have no competing interests.

\section{References}

1. The Republic of Uganda, Uganda Population-based HIV Impact Assessement, UPHIA 2016-2017. 2017.

2. Republic of Uganda, AIDS Indicator Survey (AIS), 2011. 2011.

3. Republic of Uganda, Uganda HIV/AIDS country progress report July 2016-June 2017. 2017.

4. UNAIDS, UNAIDS DATA 2018; State of the Epidemic 2018: website; http://www.unaids.org/sites/default/files/media_asset/unaids-data-2018_en.pdf.

5. Wabwire-Mangen, F., et al., HIV modes of transmission and prevention response analysis. Kampala, Uganda National AIDS Commission, 2009.

6. Hladik, W., et al., Burden and characteristics of HIV infection among female sex workers in Kampala, Uganda-a respondent-driven sampling survey. BMC public health, 2017. 17(1): p. 565.

7. Muldoon, K.A., A systematic review of the clinical and social epidemiological research among sex workers in Uganda. BMC public health, 2015. 15(1): p. 1226.

8. Vandepitte, J., et al., HIV and other sexually transmitted infections in a cohort of women involved in high risk sexual behaviour in Kampala, Uganda. Sexually transmitted diseases, 2011. 38(4): p. 316.

9. Vandepitte, J., et al., Prevalence and correlates of Mycoplasma genitalium infection among female sex workers in Kampala, Uganda. Journal of Infectious Diseases, 2011. 205(2): p. 289-296.

10. Fleming, D.T. and J.N. Wasserheit, From epidemiological synergy to public health policy and practice: 
the contribution of other sexually transmitted diseases to sexual transmission of HIV infection. Sexually transmitted infections, 1999. 75(1): p. 3-17.

11. Wawer, M.J., et al., Rates of HIV-1 transmission per coital act, by stage of HIV-1 infection, in Rakai, Uganda. The Journal of infectious diseases, 2005. 191(9): p. 1403-1409.

12. Freeman, E.E., et al., Herpes simplex virus 2 infection increases HIV acquisition in men and women: systematic review and meta-analysis of longitudinal studies. Aids, 2006. 20(1): p. 73-83.

13. World Health Organization. Delivering HIV Test Results and Messages for Re-Testing and Counselling in Adults. Geneva, Switzerland: WHO Library Cataloguing-in-publication Data; 2010. . 2010; Available from: https://apps.who.int/iris/bitstream/handle/10665/44278/9789241599115_eng.pdf?sequence=1.

14. World Health Organization. Prevention and treatment of HIV and otHer Sexually TranSmitted Infections for Sex Workers in Low and Middle-Income Countries: Recommendations for a public health approach. 2012; Available from:

https://apps.who.int/iris/bitstream/handle/10665/77745/9789241504744_eng.pdf?sequence=1.

15. World Health Organization, Policy brief: Consolidated guidelines on HIV prevention, diagnosis, treatment and care for key populations. 2017, World Health Organization.

16. Denison, J.A., et al., HIV voluntary counseling and testing and behavioral risk reduction in developing countries: a meta-analysis, 1990-2005. AIDS and Behavior, 2008. 12(3): p. 363-373.

17. Fonner, V.A., et al., Voluntary counseling and testing (VCT) for changing HIV-related risk behavior in developing countries. Cochrane database of systematic reviews, 2012(9).

18. Cohen, M.S., et al., Prevention of HIV-1 infection with early antiretroviral therapy. New England journal of medicine, 2011. 365(6): p. 493-505.

19. Wanyenze, R.K., et al., "When they know that you are a sex worker, you will be the last person to be treated": Perceptions and experiences of female sex workers in accessing HIV services in Uganda. BMC international health and human rights, 2017. 17(1): p. 11.

20. UAC. National HIV and AIDS Strategic Plan 2015/2016 - 2019/2020. Kampala, Uganda: Uganda AIDS Commission.; Available from: http://library.health.go.ug/publications/service-delivery-diseases-controlprevention-communicable-diseases/hivaids/national-h-1.

21. Nakanwagi, S., et al., Facilitators and barriers to linkage to HIV care among female sex workers receiving HIV testing Services at a Community-Based Organization in Periurban Uganda: a qualitative study. Journal of sexually transmitted diseases, 2016. 2016.

22. De Vries, H., An integrated approach for understanding health behavior; the I-change model as an example. Psychol Behav Sci Int J, 2017. 2(2): p. 10.19080.

23. Fuster-Ruizde Apodaca, M.J., et al., Psychosocial determinants of HIV testing across stages of change in Spanish population: a cross-sectional national survey. BMC public health, 2017. 17(1): p. 234. 24. Eldredge, L.K.B., et al., Planning health promotion programs: an intervention mapping approach. 2016: John Wiley \& Sons.

25. Rich, A., et al., Theory of planned behavior and adherence in chronic illness: a meta-analysis. Journal of behavioral medicine, 2015. 38(4): p. 673-688.

26. Shannon, K., et al., Global epidemiology of HIV among female sex workers: influence of structural determinants. The Lancet, 2015. 385(9962): p. 55-71. 
27. Uganda Bureau of Statistics, National population and housing census 2014. 2014, Uganda Bureau of Statistics Kampala (Uganda).

28. Batona, G., et al., Understanding the intention to undergo regular HIV testing among female sex workers in Benin: a key issue for entry into HIV care. JAIDS Journal of Acquired Immune Deficiency Syndromes, 2015. 68: p. S206-S212.

29. Santos, J.R.A., Cronbach's alpha: A tool for assessing the reliability of scales. Journal of extension, 1999. 37(2): p. 1-5.

30. Trujillo-Ortiz, A., et al., kmo: Kaiser-Meyer-Olkin Measure of Sampling Adequacy. A MATLAB file.[WWW document]. URL http://www. mathworks. com/matlabcentral/fileexchange/loadFile. do, 2006.

31. Tobias, S. and J.E. Carlson, Brief report: Bartlett's test of sphericity and chance findings in factor analysis. Multivariate Behavioral Research, 1969. 4(3): p. 375-377.

32. Sullivan, G.M. and A.R. Artino, Jr., Analyzing and interpreting data from likert-type scales. J Grad Med Educ, 2013. 5(4): p. 541-2.

33. Norman, G., Likert scales, levels of measurement and the "laws" of statistics. Adv Health Sci Educ Theory Pract, 2010. 15(5): p. 625-32.

34. Musyoki, H., et al., Prevalence of HIV, sexually transmitted infections, and risk behaviours among female sex workers in Nairobi, Kenya: results of a respondent driven sampling study. AIDS and Behavior, 2015. 19(1): p. 46-58.

35. Ingabire, R., et al., Female sex workers in Kigali, Rwanda: a key population at risk of HIV, sexually transmitted infections, and unplanned pregnancy. International journal of STD \& AIDS, 2019: p. 0956462418817050.

36. Uganda AIDS Commission, National HIV and AIDS Strategic Plan 2015/2016 - 2019/2020. 2015. 37. Ajzen, I., The theory of planned behavior. Organizational behavior and human decision processes, 1991. 50(2): p. 179-211.

38. Ogilvie, G.S., et al., Correlates of women's intentions to be screened for human papillomavirus for cervical cancer screening with an extended interval. BMC public health, 2016. 16(1): p. 213.

39. Ebu, N.I. and J.K. Ogah, Predictors of cervical cancer screening intention of HIV-positive women in the central region of Ghana. BMC women's health, 2018. 18(1): p. 43.

40. Abamecha, F., A. Tena, and G. Kiros, Psychographic predictors of intention to use cervical cancer screening services among women attending maternal and child health services in Southern Ethiopia: the theory of planned behavior (TPB) perspective. BMC public health, 2019. 19(1): p. 434.

41. Muhindo, R., A. Nakalega, and J. Nankumbi, Predictors of couple HIV counseling and testing among adult residents of Bukomero sub-county, Kiboga district, rural Uganda. BMC public health, 2015. 15(1): p. 1171.

42. Allom, V., et al., Physical activity and transitioning to college: The importance of intentions and habits. American journal of health behavior, 2016. 40(2): p. 280-290.

43. Triandis, H.C. Values, attitudes, and interpersonal behavior. in Nebraska symposium on motivation. 1979. University of Nebraska Press.

44. Ministry of Health, Consolidated Guidelines for the Prevention and Treatment of HIV and AIDS in Uganda. 2018. 
45. Nnko, S., et al., Determinants of access to HIV testing and counselling services among female sex workers in sub-Saharan Africa: a systematic review. BMC public health, 2019. 19(1): p. 15.

46. Chanda, M.M., et al., Barriers and facilitators to HIV testing among Zambian female sex workers in three transit hubs. AIDS patient care and STDs, 2017. 31(7): p. 290-296.

47. Wong, W.C.-W., et al., Evaluation and mechanism analysis of HIV prevention programme using resilience framework among female sex workers: A randomised controlled trial. Preventive medicine reports, 2019. 13: p. 229-237.

48. Oldenburg, C.E., et al., Zambian Peer Educators for HIV Self-Testing (ZEST) study: rationale and design of a cluster randomised trial of HIV self-testing among female sex workers in Zambia. BMJ open, 2017. 7(4): p. e014780.

49. Rowe, J.A., REVOLUTION IN BUGANDA 1856-1900. PART ONE: THE REIGN OF KABAKA MUKABYA MUTESA 1856-1884. 1968.

\section{Tables}

Due to technical limitations, all tables are only available for download from the Supplementary Files section.

\section{Supplementary Files}

This is a list of supplementary files associated with this preprint. Click to download.

- DatacollectiontoolQuestionnaire.docx

- Tables.pdf 